\title{
The evaluation of strings in an artificial logical language'
}

WILLIAM J. KROSSNER ${ }^{2}$

HARVARD UNIVERSITY

Ss given the rule specifying the well-formedness of logical propositions written in Lukasiewicz parenthesis-free notation were required to judge an array of well-formed and non-well-formed strings as to their status. Times to solution were positively correlated with errors, and were monotonically dependent on string length and on a function related to the degree of embedding in the string.

Formal languages such as those of mathematics and logic possess certain features which have analogues in natural languages. The notions of grammaticalness and well-formedness form one pair of such analogues. An array of words put together according to the rules of English grammar is said to be a grammatical sentence; a set of symbols put together according to the formation rules of a formal language is said to be a well-formed string. In both cases, rules specify which sequences of symbols are permissible in the language and which are prohibited.

Since the grammar of a natural language possesses great complexity (Chomsky, 1964), the study of rule governed processes may occasionally be shifted away from natural language itself to systems that possess simpler formation rules, both because such systems may be interesting in their own right, and because their analysis may possibly shed light on the more difficult problems of natural language usage. A particularly simple formal language is given by the parenthesis-free notation system of Lukasiewicz (1951) that permits the writing of compound logical forms of the propositional calculus without punctuation symbols, and which is the basis of the game of WFF'N PROOF (Allen, 1962).

Miller (1967) has reported an experiment of Norman and Schneider in which a computer presented examples of Lukasiewicz strings three symbols in length to Ss who attempted to learn the rule of formation inductively. This task proved too difficult; most Ss could not learn the rule in sessions lasting $2 \mathrm{~h}$. In contrast, the present experiment did not ask the Ss to discover the rule inferentially; rather, it involved presenting the rule to the $\mathrm{Ss}$ in advance, and testing their ability to use it to evaluate strings as to well-formedness.

Burks, Warren, \& Wright (1954) presented the theoretical analysis of Lukasiewicz notation in terms of the necessary features a conceptual automaton must possess if it were to have the capability to evaluate this language. An essential determinant of the complexity of the automaton is a property of the strings to be analyzed known as the "maximum tail weight," hereafter in this paper to be called simply "tail weight." (Care should be taken not to confuse this nomenclature with that of Burks, Warren, and Wright, who reserve the term "tail weight" for more general usage.) Strings with a high tail weight have the constituent well-formed parts of the string embedded in each other so that the analysis of a constituent must proceed simultaneously with the analysis of all embedded constituents until a symbol is reached which jointly serves as a terminating element.

In view of these results, it was hypothesized that Ss familiar with the rule of formation for Lukasiewicz strings would require time to analyze strings for well-formedness as a monotonically increasing function both of the number of symbols comprising the string (the string length) and of the measure of string complexity (the tail weight).

\section{Subjects}

Twenty-seven students from the Harvard Summer School were paid Ss.

\section{Materials and Procedure}

The symbols A, K, N, p, q, r were used as the characters. A set of 17 well-formed strings using these symbols was constructed, with tail weights in the set ranging from 2 through 6 , and string lengths ranging from 6 symbols through 15. A set of 17 nonwell-formed strings, matched for length, was also constructed, as well as a set of 13 practice problems whose tail weight did not exceed 2 and whose length did not exceed 7. Each string was printed on an index card.

Ss, run individually, received an instruction sheet that contained the formation rule for Lukasiewicz strings and were allowed to keep the sheet to refer to the rule at any time. 4 They were instructed to examine a string for as long a time period as they needed, and to classify it as correct, i.e., conforming to the rule (well-formed), or incorrect.

Ss first recelved the practice problems to ascertain that they understood the rule and to absorb the considerable warmup effect to be expected in any reaction time procedure. Practice problems were divided between well-formed and non-well-formed strings and were mainly of very short length. The practice problems with a length greater than 4 were included to ensure that Ss realized that the formation rule permitted strings of any length to be possibly correct. Data obtained from the practice problems were discarded. After the practice problems, the 34 
Table 1. Harmonic Mean Solution Times, Seconds

\begin{tabular}{lccccccc} 
& & \multicolumn{7}{c}{ String } & Length \\
& & 6 & 8 & 10 & 12 & 13 & 15 \\
\hline Well-Formed: & 2 & 5.66 & 6.79 & 10.88 & 16.22 & 17.05 & \\
Tail Weight & 3 & 6.68 & 8.76 & 11.86 & 17.44 & 19.14 & \\
& 4 & & & 16.13 & 18.82 & 18.85 & \\
& 5 & & & & 19.52 & 19.81 & 22.19 \\
& 6 & & & & & & 26.16 \\
\hline Non-well- & & 6.36 & 7.68 & 12.40 & 16.68 & 20.39 & 24.92 \\
Formed & & & & & & & \\
\hline
\end{tabular}

problems of the main sequence were presented in an order that was rerandomized for each $\mathrm{S}$.

E measured the time from the presentation of a string to S's response with a Standard Electric timer, and recorded both time and response.

Results and Discussion

The times obtained from Ss were subjected to the reciprocal transformation to reduce the possible skewing effects of any long reaction times (Edwards, 1950) and harmonic means were computed. Table 1 presents means based on correct answers; times associated with incorrect answers were not used as components of those means on the grounds that, since the answer was wrong, $\mathbf{S}$ must have used an incorrect and unknown process in analyzing the string. With only one exception (19.14 sec for string length 13), the means of Table 1 lie in the correct monotonic order to confirm both original hypotheses. An increase in either tail weight or string length produced an increase in mean solution time. The means for the non-well-formed strings, classified only by length since the concept of tail weight has little relevance for strings which may be non-well-formed in an infintte number of ways, provide confirming evidence as to the effect of length on solution time.

Obtaining an overall statistical test of trend for the data of Table 1 poses a formidable problem because of missing cell entries and the fact that the row and column entries are correlated measures based on a common population of Ss. The missing cell entries were necessitated by the nature of the material; it is impossible, for example, to construct a well-formed string of tail weight 6 with only 8 symbols. However, as an approach to testing significance, Kendall's $S$ was computed across Ss for each of the four possible individual rows and each of the six columns. The $S$ computations necessarily used solution times for both right and wrong answers, and each $S$ by itself was significant $(p<.05)$, strongly implying the overall significance of both tail weight and string length.

The overall error rate for well-formed strings was 16.8\%; the Spearman rho coefficient of correlation between times and errors was .47 . The overall error rate for the non-well-formed strings was $13.0 \%$.

To the extent that tail weight can be considered the analogue of linguistic embedding, the result of the present experiment that a string with a higher tail weight is a more complex string is in agreement with that of Miller \& Isard (1964), who showed that increasing the number of degrees of embedding in sentences made the sentences more difficult to remember. The present results also suggest that the study of other systems in mathematics and logic may yield further analogies between these systems and natural language.

References

ALLEN, L. E. WFF'N PROOF: The game of modern logic. New Haven: Privately printed, 1962.

BURKS, A. W., WARREN, D. W., \& WRIGHT, J. B. An analysis of a logical machine using parenthesis-free notation. Mathematical tables and other aids to computation, 1954, 8, 53-57.

CHOMSKY, N. A spects of the theory of syntax. Cambridge, Mass.: MIT Press, 1965.

EDWARDS, A. E. Experimental design in psychological research. New York: Rinehart, 1950.

LUKASIEWICZ, J. Aristotle's syllogistic from the standpoint of moder formal logic. Oxford: Clarendon Press, 1951.

MILLER, G. A. The psychology of communication. New York: Basic Books, 1967.

MILLER, G. A., \& ISARD, S. Free recall of self-embedded English sentences. Information and Control, 1964, 7, 292-303.

Notes

1. This research was supported by Contract SD-187 from the Department of Defense, Advanced Research Projects Agency, to Harvard University, Center for Cognitive Studies. Thanks are due to Dr. A. R. Jonckheere for help with the statistical analysis.

2. Now at Department of Psychology, Vassar College, Poughkeepsie, New York.

3. Maximum tail weight may be computed as follows: Assign weight -1 to operators $A$ and $K$, representing disjunction and conjunction respectively, weight 0 to the operator $N$, representing negation, and weight 1 to the propositional variables $p, q$, r. On a pass through the string from right to left, compute the sum of weights symbol by symbol until the beginning of the string is reached. The highest value attained is the maximum tail weight. Thus, the string AApNrq has a maximum tail weight of 3 . A string is well-formed if and only if the sum of weights during a pass always remains positive and the weight of the entire string is 1 . Thus, $\mathrm{KqrNp}$ is not well-formed.

4. The rule was, "A sequence of letters is correct if, and only if, (1) It is a p, q, or r, OR (2) it begins with $\mathrm{N}$ and is followed by a correct sequence, OR (3) it begins with $A$ or $K$ and is followed by two correct sequences." 